\title{
An Enhancement to the Constant Range Method for Nonuniformity Correction of Infrared Image Sequences ${ }^{\star}$
}

\author{
Jorge E. Pezoa, Sergio N. Torres, Juan P. Córdova, and Rodrigo A. Reeves \\ Department of Electrical Engineering, University of Concepción \\ Casilla 160-C, Concepción, Chile \\ \{jpezoa, storres\}@die.udec.cl \\ jcordova@udec.cl \\ rreeves@die.udec.cl \\ http://nuc.die.udec.cl/
}

\begin{abstract}
A statistical technique for adaptive nonuniformity correction of infrared image sequences has been developed. The method, which relies on our previously developed constant range nonuniformity correction method, estimates the nonuniformity parameters using two recursive estimation techniques. The method selects an estimation algorithm using a decision rule based on a threshold value computed from the collected infrared images. The strength of the method lies in its simplicity, low computational complexity, and its good trade-off between nonuniformity correction and ghosting artifacts reduction. The ability of the enhanced constant range technique to compensate for nonuniformity noise is demonstrated by using video sequences of infrared imagery with both real and synthetic nonuniformity.
\end{abstract}

\section{Introduction}

Since the 1970's, infrared (IR) imaging technology has proven to be an invaluable tool in a wide range of industry, medical, and military applications. IR cameras have been used for temperature measurements, IR signature analysis, tracking applications, medical and military usage, stress measurements, detection and prediction of failures modes within machinery, etc. For several technical and economical reasons, the focal plane array (FPA), an integrated circuit containing an array of IR photodetectors, is the most commonly used sensor technology employed in IR cameras [1].

However, the performance of the whole imaging system is strongly affected by the main disadvantage of the FPA: the random spatial response of each

\footnotetext{
^ This work was partially supported by the 'Fondo Nacional de Ciencia y Tecnología' FONDECYT of the Chilean government, project number 1020433 and by Grant Milenio ICM P02-049. The authors wish to thank Ernest E. Armstrong (OptiMetrics Inc., USA) for collecting the data, and the United States Air Force Research Laboratory, Ohio, USA.
} 
individual photodetector in the array under the same uniform input $[1,2]$. This nonuniformity (NU), produced by mismatches during the fabrication process of the IR sensor, can considerably degrade the quality of IR images, since it results in a fixed-pattern-noise $(\mathrm{FPN})$ that is superimposed on the true image. Furthermore, the problem is made worse because the NU is not totally stationary, but instead it drifts slowly and randomly over time. This temporal drift can take, from minutes to hours, depending on the technology used.

It is well known that the NU correction (NUC) of IR images is a great advantage for any kind of post processing analysis such as pattern recognition, image restoration, image registration, etc. [3]. To compensate for the NU, two categories of NUC methods have been developed: calibration-based and scenebased NUC methods. Calibration-based methods need to steer the FPA away from the target to an uniform black-body radiation source used as a reference. This is very unlikely in real time applications; it forces the interruption of the normal imaging operation. On the other hand, scene-based techniques require image sequences with motion or changes in the actual scene to be able to perform the NUC. If the motion requirement is not fully satisfied, these approaches produce a lower quality NUC than calibration-based techniques. In addition, when an object does not move enough, a ghost image of itself, also called a ghosting artifact, can be observed over the corrected IR images even when the object has left the field of view $[1,2]$.

In this paper, an extension of our previously developed constant range (CR) scene-based NUC method is presented [4]. The enhanced CR (ECR) improves the CR method by adding two extra features: an exponential windowing (EW) estimation algorithm, and a decision criteria to adaptively change the estimation algorithm. Read-out mean and variance are recursively estimated with an EW algorithm, which converges faster than the one used in the CR method. The EW algorithm produces a faster update for the estimation because it weights the recent data most heavily; however, it also produces an oscillating effect over the estimation. Changing between the traditional CR's estimation method and the EW algorithm is decided by thresholding the incoming read-out frames. Thus, the ECR NUC method obtains a good trade-off between the speed and the quality of the estimation. As a result, these features naturally can help reduce the ghosting artifacts produced by the scene-based NUC. To test the scheme, the ECR technique is applied to sequences of IR images with both simulated and real NU and the performance of the scheme is tested using the root mean square error (RMSE) between the corrected and the uncorrected frames and the image quality parameter $Q$-factor [5].

This paper is organized as follows. Section 2 presents the linear model for an FPA and the CR NUC method. Also, the ECR NUC method is introduced. In Section 3 the ECR algorithm is tested with sequences of IR data with both, simulated and real NU, and the performance parameters are computed. The conclusions of the paper are summarized in Section 4. 


\section{The Enhanced Constant Range Nonuniformity Correction Method}

In this section the mathematical models of an IR-FPA and the CR NUC method are presented. Using this background, the ECR algorithm is developed and theoretical calculations about its performance are derived.

\subsection{The Mathematical Model of an Infrared Focal Plane Array}

Most NUC methods consider, for a single operating point, the linear model given in [1]. In this model, the mathematical relationship between the read-out IR data $Y_{k}^{i j}$ in the $i j-t h$ detector of the FPA, and at time $k$, with the input irradiance $T_{k}^{i j}$ can be written as:

$$
Y_{k}^{i j}=A^{i j} T_{k}^{i j}+B^{i j}
$$

where $A^{i j}$ and $B^{i j}$ are the gain and bias for $i j$-th detector in the array, respectively. $A^{i j}$ and $B^{i j}$ can be modelled as constants when no significant drift on the NU is considered and this condition is satisfied capturing a couple of minutes of data. Finally, the input $T_{k}^{i j}$ represents the irradiance collected at the $k$-th frame time by the $i j$-th IR detector of the FPA. For simplicity of notation, the pixel superscripts ij will be omitted with the understanding that all operations are performed on a pixel-by-pixel basis.

\subsection{The Constant Range Algorithm}

The task of any NUC method is to estimate $A$ and $B$ using the information obtained from $Y_{k}$. To achieve this estimation, additional information is required, therefore, assumptions about $T$ are necessary. The key assumption in the CR method is that the input irradiance is an uniformly distributed random variable within each sequence of frames and all detectors are exposed to, approximately, the same range of input irradiance. Assuming a common range $\left[T_{\min }, T_{\max }\right]$, it allows us to estimate the gain and the bias for each pixel to within unknown but global scale and offset factors. So, $T_{\min }$ and $T_{\max }$ can be considered known parameters, and they depend only on the camera used to collect the IR information [4]. Finally, this condition can be easily met, for example, in the presence of motion.

Using the CR assumption, the method estimates the gain and the bias based on the following equations [4]:

$$
\begin{aligned}
& \hat{A}=\frac{\hat{\sigma}_{Y}}{\hat{\sigma}_{T}} \\
& \hat{B}=\hat{m}_{Y}-\frac{\hat{\sigma}_{Y}}{\hat{\sigma}_{T}} \hat{m}_{T}
\end{aligned}
$$

where $\hat{m}_{T}\left(\hat{\sigma}_{T}\right)$ and $\hat{m}_{Y}\left(\hat{\sigma}_{Y}\right)$ are the estimated expected values (the standard deviation) of $T$ and $Y$, respectively. In [4], $\hat{m}_{T}$ and $\hat{\sigma}_{T}$ are estimated a priori with the CR assumption, and $\hat{m}_{Y}$ and $\hat{\sigma}_{Y}$ are recursively estimated using: 


$$
\begin{aligned}
\hat{m}_{Y, k} & =\frac{Y_{k}+(k-1) \hat{m}_{Y, k-1}}{k} \\
\hat{\sigma}_{Y, k} & =\frac{\left|Y_{k}-\hat{m}_{Y, k}\right|+(k-1) \hat{\sigma}_{Y, k-1}}{k}
\end{aligned}
$$

\subsection{The Proposed Enhancement on the Constant Range Method}

The system model (1) considers the gain and the bias as stationary-unknown parameters. But, it was stated that the NU changes slowly and randomly over time. Therefore, the estimation provided by any NUC method must follow this drift. In [2], the following EW recursive estimation algorithm for $\hat{m}_{Y}$ and $\hat{\sigma}_{Y}$ is presented:

$$
\begin{aligned}
\hat{m}_{Y, k} & =(1-\alpha) Y_{k}+\alpha \hat{m}_{Y, k-1} \\
\hat{\sigma}_{Y, k} & =(1-\alpha)\left|Y_{k}-\hat{m}_{Y, k}\right|+\alpha \hat{\sigma}_{Y, k-1}
\end{aligned}
$$

where $\alpha, 0<\alpha<1$, is the time constant of the filter that controls the exponential window of the read-out data. Because an EW algorithm emphasizes recent data, it provides the NUC method with the ability to follow changes in the operating point and, besides, it helps the algorithm to reduce ghosting artifacts [2]. However, a faster convergence of the estimator may reduce the quality of the NUC.

The ECR NUC method computes $\hat{m}_{Y}$ and $\hat{\sigma}_{Y}$ using either $(4,5)$ or $(6,7)$ equations. It selects which estimation algorithm to use based on a threshold value. The algorithm detects the changes, per pixel, between the current and the previous read-out data, and it compares the difference with the threshold. If the change is greater than the threshold, then the ECR employs the EW estimation algorithm to accelerate the convergence of the estimation. On the other hand, if no significative change in $Y_{k}$ is observed, the algorithm $(4,5)$ has been shown to yield a good estimation [4]. Furthermore, in real situations, the difference between consecutive frames is very low due to the sample rate of the camera; then, the threshold comparison is performed between the $k$-th and the $k+\Delta k$-th frames. It can be seen from the foregoing analysis that only two parameters of the algorithm need to be tuned: the threshold value and the stride value $\Delta k$.

Finally, the ECR algorithm does not require a great computing time effort; it only needs fourteen additions, fourteen multiplications and five logical instructions, for each pixel at each frame.

\section{Performance Analysis}

In this section the ECR NUC method is tested using sequences of IR images corrupted with simulated and real NU. The NUC performance is evaluated using the performance indexes RMSE and $Q$-factor [5] computed between the reference (an IR sequence calibrated with black bodies) and the corrected IR video sequence. For the $Q$-factor, the dynamical range is $[-1,1]$, where +1 represents the best 
performance [5]. The sequence used during the tests is a video of terrestrial midwave IR imagery $(3 \sim 5 \mu \mathrm{m})$ collected with a $128 \times 128 \mathrm{InSb}$ FPA cooled camera (Amber Model AE-4128). The video contains 4000 frames captured at a rate of $30 \mathrm{fps}$, and each pixel is quantized in 16-bit integers: $\left[Y_{\min }, Y_{\max }\right]=[0,65535]$. In addition, the sequence was corrected using calibration-based techniques and will be used as reference when needed.

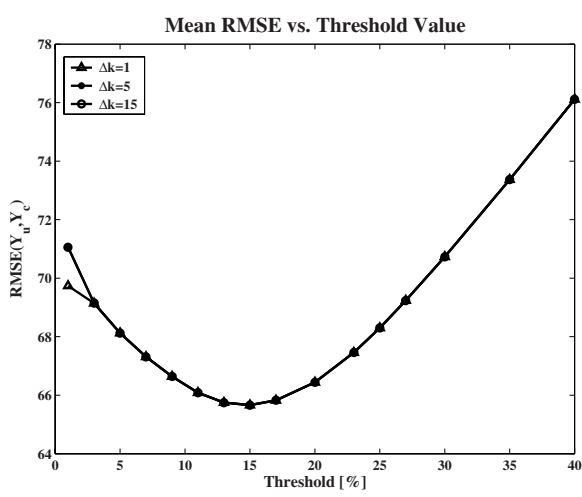

a)

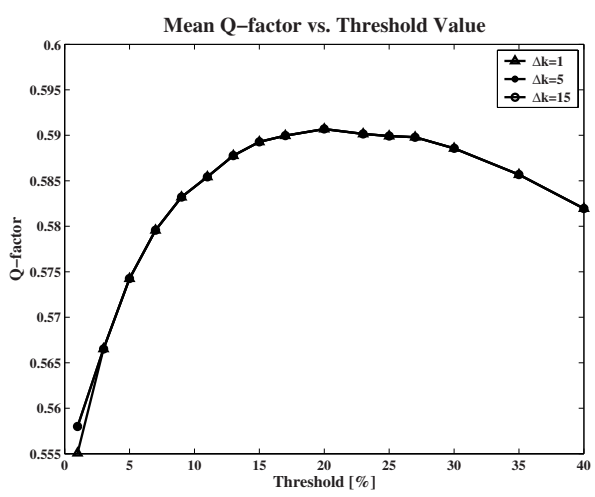

b)

Fig. 1. Performance of the NUC methods under simulations. a) The mean RMSE for the sequence of IR data vs. the threshold value. b) The mean $Q$-factor for the sequence of IR data vs. the threshold value. (The $\triangle$ sign means a stride value $\Delta k=1$. The $\bullet$ sign means a stride value $\Delta k=5$. The $\circ$ sign means a stride value $\Delta k=15$.)

In all simulations, the gain and the bias are considered as spatially and mutually uncorrelated Gaussian random variables with mean values of one and zero, respectively. Different levels of nonuniformity are introduced by varying the standard deviation of the gain and the bias. Finally, all our simulations were made following the standard procedures given in $[2,4]$.

The ECR algorithm depends on the following quantities: the threshold value and the stride parameter $\Delta k$. To tune these values, the ECR method was applied to a video of frames with simulated NU. Threshold values between 1 and $40 \%$ of $Y_{\max }$ were tested for the following strides: $\Delta k=\{1,2,3,4,5,8,10,12,15\}$. For the EW estimation algorithm used in the ECR method, the $\alpha$ time constant of the filter was established to be 0.99 , in accord to the results obtained in [2]. Figs. 1a) and b) plot the results for the mean RMSE and the mean $Q$-factor of the video of frames vs. the threshold values considered, for the stride values of 1, 5, and 15 frames. The results show that a good choice for the threshold is between 15 and $20 \%$ of the maximum quantization value used by the IR camera. Further, our computations demonstrate that no significant enhancement in the RMSE and the Q-factor is obtained for the stride values evaluated.

Using the previous results, the ECR NUC method was tested with a new sequence of IR data also corrupted with synthetic NU. The threshold value and 
the stride value of the ECR were chosen as $15 \%$ of $Y_{\max }$ and 1 frame, respectively. In Figs. 2b), c), d) and e) an example of the real, the corrupted and the corrected IR images obtained with simulations can be observed. It can be noted that, to the naked eye, the ECR produces a better NUC than the CR method. Again, a visual inspection shows that the image corrected with the ECR algorithm is less affected by the ghosting than the one corrected with the CR method. A subjective evaluation, computed with the performance parameters RMSE and $Q$-factor, agrees also with the objective evaluation. Fig. 3a) and b) illustrate the time evolution of these parameters. The ECR method produces a lower RMSE than the CR method. Furthermore, the ECR's RMSE is lower than the one obtained with a CR method that utilizes an EW estimation algorithm instead of the traditional algorithm $(4,5)$. The same behavior is seen when the $Q$-factor is computed: the ECR NUC method achieves the best values. In addition, a closer look at Figs. 2d) and e) shows a ghost image of the Fig. 2a) imposed over the corrected images. However, it can also be noted that the proposed algorithm produces less ghosting artifacts than the CR method.

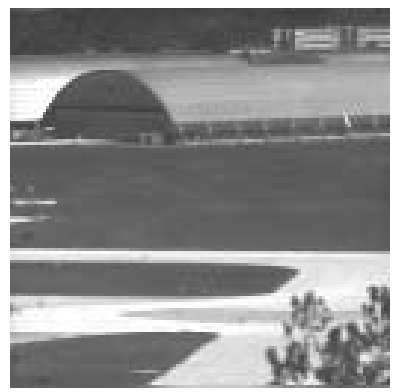

a)

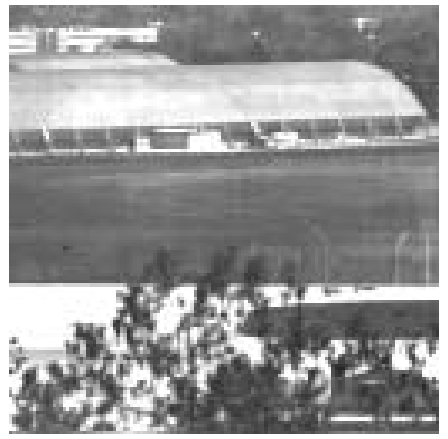

d)

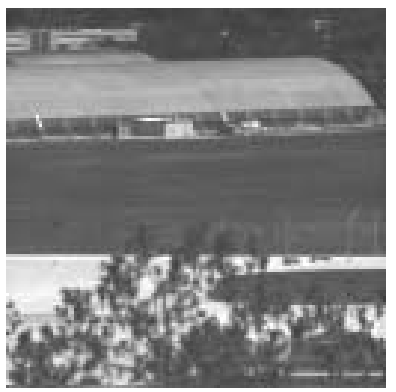

b)

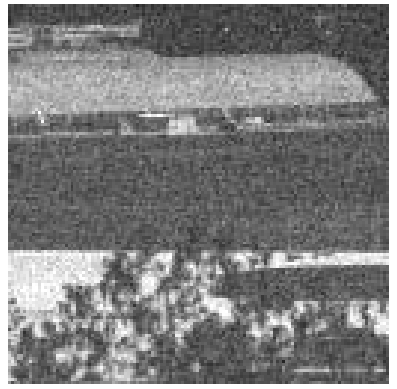

c)

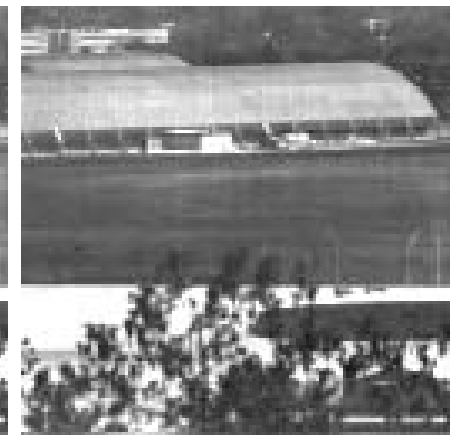

e)

Fig. 2. Performance of the ECR NUC method under simulations. a) The 1560-th frame of the reference IR image sequence b) The 1680-th frame of the reference c) The 1680th frame corrupted with synthetic NU d) The 1680-th frame corrected using the CR method e) The 1680-th frame corrected using the ECR method. Note in images d) and e) the effect of the ghosting artifact introduced by the 1560-th frame. 


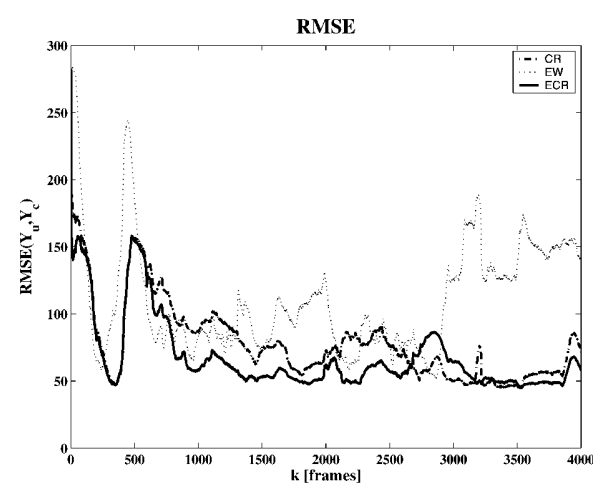

a)

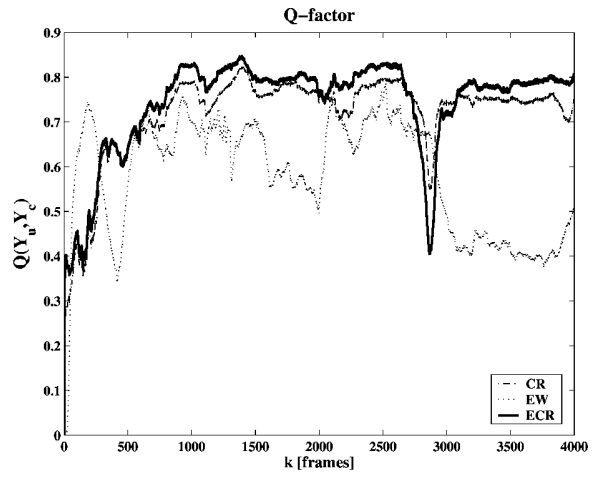

b)

Fig. 3. Performance of the NUC methods under simulations. a) The evolution of the RMSE between the reference and the corrected frames of IR data. b) The evolution of the $Q$-factor between the reference and the corrected frames of IR data (CR means constant range NUC method. EW means CR with exponential windowing. ECR means enhanced constant range).

A block of frames with real NU was also used to test the performance of the ECR NUC method. According to the previous simulations and after several tests, we obtain the best results for the following parameters: $\alpha=0.99$, a threshold value of $17 \%$ of $Y_{\max }$, and $\Delta k=3$. Figs. 4a) and d) show examples of the raw frame captured by the camera. Figs. 4b) and e) are their corresponding frames corrected using the CR method, and Figs. 4c) and f) are corrections made with the ECR algorithm. The results show that the ECR method effectively compensates the NU and produces, to the naked eye, a better NUC than the $\mathrm{CR}$ algorithm. Similar results are obtained for the performance parameters. As an example, the computed RMSE of the 2280-th frame for the CR method is $0.123 \%$ of $Y_{\max }$, whereas the ECR algorithm obtains a $0.064 \%$. The computed $Q$-factor for this frame are 0.607 and 0.668 for the CR and the ECR method, respectively. Besides, the images in Fig. 4b) and c) show that the ECR method produces less ghosting artifacts than the CR method. Finally, it can be seen from Fig. 4 that the proposed method inherits the valuable CR ability of compensating for malfunctioning pixels.

\section{Conclusions}

In this paper an enhanced version of our previously developed constant range nonuniformity correction method is proposed. The algorithm estimates the gain and the bias of the infrared sensors with two estimation techniques and, using the read-out data, adaptively selects which one to employ. The method is quite simple, utilizes low computational resources and needs to tune only two parameters. Our evaluations, using infrared video sequences corrupted with both real 


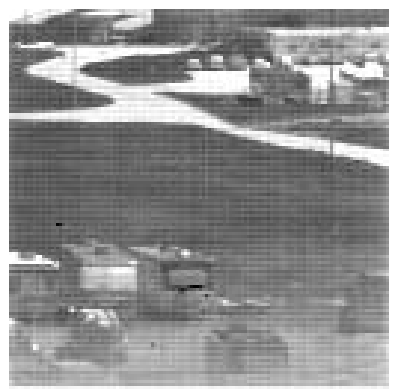

a)

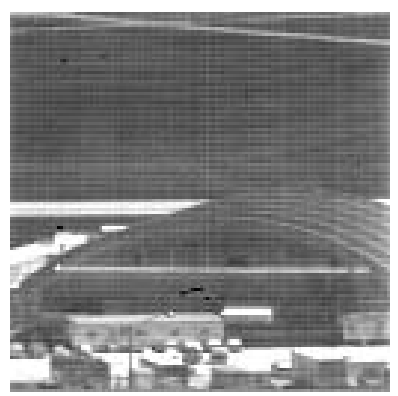

d)

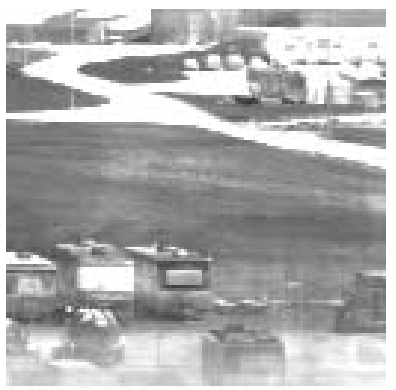

b)

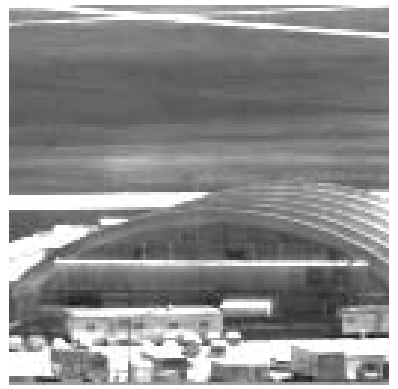

e)

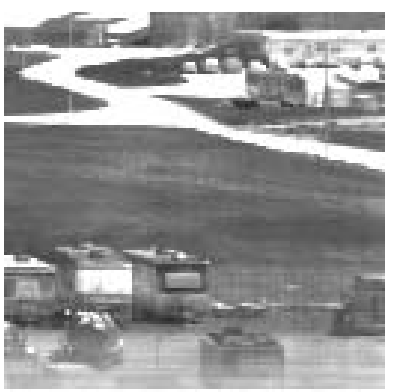

c)

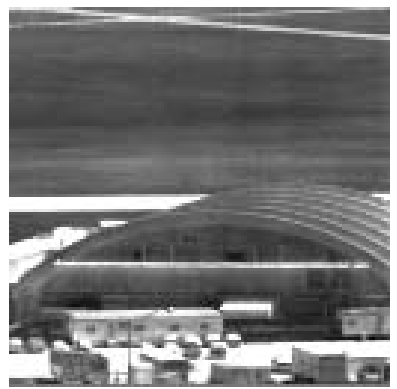

f)

Fig. 4. Performance of the ECR NUC method under real NU. a) The 2280-th frame with real NU b) The 2280-th frame corrected with the CR method c) The 2280-th frame corrected with the ECR method d) The 2680-th real frame e) The 2680-th frame corrected using the CR method f) The 2680-th frame corrected using the ECR method.

and simulated nonuniformity, have shown that the approach not only performs an efficient nonuniformity correction of the sequences, but also it produces compensated images with better quality than the original constant range method.

\section{References}

1. Holst, G.: CCD arrays, cameras and displays. SPIE Optical Engineering Press. Bellingham. (1996).

2. Harris, J., Chiang, Y-M.: Nonuniformity Correction of Infrared Image Sequences Using the Constant-Statistics Constraint. IEEE Transactions on Image Processing. 8. (1999) 1148-1151.

3. Armstrong, E., Hayat, M., Hardie, R., Torres, S., Yasuda, B.: The Advantage of NonUniformity Correction Pre-Processing on Infrared Image Registration. Application of Digital Image Processing. XXII. (1999).

4. Torres, S., Reeves, R.: Scene-Based Nonuniformity Correction Method Using Constant-Range. Performance and Analysis. Proc. SCI 2002/ISAS 2002. IX. (2002).

5. Wang, Z., Bovik, A.: A Universal Image Quality Index. IEEE Signal Processing Letters. 20. (2002) 1-4. 